\title{
Estrategias, estilos de aprendizaje y rendimiento académico en estudiantes ingresantes de Odontología
}

\author{
Strategies, learning styles and academic performance in \\ undergraduate students of dentistry
}

\begin{abstract}
Resumen
La Facultad de Odontología está comprometida en la búsqueda de la calidad educativa, lo que implica que el docente en su ejercicio académico debe propiciar el aprendizaje de los estudiantes; interactuando con el conocimiento a través de actividades variadas, que atiendan las diferencias individuales en relación a sus formas, estilos y estrategias de aprender para un mejor rendimiento académico. El presente estudio tiene como objetivo identificar las estrategias, los estilos de aprendizaje en los estudiantes ingresantes en la Facultad de Odontología y su relación con el rendimiento académico. Las unidades de observación lo constituyen 26 estudiantes que participaron en forma voluntaria en la convocatoria al Programa de Tutoría, el que tuvo dificultades de implementación. Se evaluó los estilos y estrategias de aprendizaje utilizando los cuestionarios de Honey-Alonso, (CHAEA) y el de las estrategias de aprendizaje de los estudiantes universitarios por Gargallo; mientras que el rendimiento académico se evaluó con el historial académico. Se halló diferencias significativas entre la motivación extrínseca inicial y final; asimismo entre la motivación con el rendimiento académico; se determinó que las estrategias se modifican y se plasman en su rendimiento; lo que describe que el estudiante ingresante aún está en proceso de construcción de su autonomía y requiere orientación.

Palabras Claves: Odontología; Estilos de aprendizaje; Rendimiento académico; Programa de tutoría.

\section{Abstract}

The Faculty of Dentistry is committed to the pursuit of quality education, which implies that the learning of the students should be promote by the teachers; they should interact with knowledge through varied activities that meet the individual differences in their relationship with their modes, styles and learning strategies in order to improve their academic performance. This study aims to identify the strategies and learning styles of the incoming students at the Faculty of Dentistry and its relationship to academic performance. Twenty-six students participated voluntarily in the Tutoring Program, this issue had implementation difficulties. Styles and learning strategies were evaluated using questionnaires: Honey-Alonso Learning Styles (CHAEA) and the learning strategies of university students by Gargallo; while academic performance was assessed with the academic record. It was found significant differences between the initial and final extrinsic motivation; also between motivation and academic performance; it was determined that strategies are modified and reflected in their academic performance. It was concluded the incoming student is still in the process of establishing their independence and requires guidance.
\end{abstract}

Keywords: Dentistry; Learning styles; Academic performance; Tutoring program.

\section{Introducción}

En estas dos últimas décadas se está produciendo un crecimiento exponencial del conocimiento, lo que nos acerca a la necesidad de estar abiertos al aprender $\mathrm{y}$ al desaprender; ser accesibles a nuevos conceptos o ideas es hoy una necesidad. El desaprender significa reconocer que lo que se ha hecho hasta un determinado momento no ha dado el resultado esperado y que es tiempo de encontrar otras formas de aprender basados en evidencias para aprender nuevamente; genera cambios de paradigmas, lo que implica hacer un esfuerzo personal para innovar. Por lo tanto, la capacidad para aprender de los individuos es un factor importante para alcanzar logros, tanto individual como colectivo, asimismo en lo académico como en lo personal y profesional.

En nuestro país, se está visibilizando altos índices de deserción, abandono y/o repitencia de los estudiantes universitarios en los primeros años de estudios; es un reto a enfrentar teniendo en cuenta el número y la heterogeneidad de los estudiantes; se requiere que los discentes sean atendidos en sus particularidades, demandan de apoyo en su proceso de

\section{Artí́culo Original}

Lita Ortiz-Fernández', Hilda MoromiNakata2 ${ }^{2}$, Carmen Quintana del Solar ${ }^{3}$, Melissa Barra-Hinostroza², Juana Bustos de la Cruz ${ }^{3}$, Lita Cáceres, Sylvia Chein-Villacampa ${ }^{1}$, Cecilia Rodríguez-Vargas ${ }^{1}$

\footnotetext{
1. Departamento Académico de Estomatología Preventiva y Social

2. Departamento Académico de Ciencias Básicas

3. Departamento Académico de Estomatología Pediátrica

1-3. Facultad de Odontología de la Universidad Nacional Mayor de San Marcos, Perú.
}

\section{Correspondencia:}

CD. Lita Ortiz Fernández

Facultad de Odontología, Universidad Nacional Mayor de San Marcos, Av. German Amezaga s/n. Lima 1, Perú.

Correo electrónico: limaoff@yahoo.es

\section{Coautores:}

Moromi-Nakata: hmnbio@hotmail.com Quintana: ortoquintana@hotmail.com Barra-Hinostroza: melisbarra@hotmail.com Bustos: cdjrbustos@yahoo.es Caceres: litacaceres@yahoo.es Chein-Villacampa: sychevi7720@hotmail.com Rodriguez-Vargas:

mceciliarodriguezv@gmail.com

Fecha de recepción: 04-06-14

Fecha de aceptación: 31-10-14 aprendizaje, implica brindarles distintas posibilidades de interacción con el conocimiento a través de actividades variadas, reconociendo las diferencias individuales en relación a sus formas, estrategias y estilos de aprender para un mejor rendimiento académico.

Hoy se hace necesario dar más atención a los procesos de aprendizaje de los estudiantes como respuesta a la demanda social de formar personas capaces y eficaces. Considerando lo referido, el aprendizaje se caracteriza por ser un proceso dinámico, continuo, global, personal y gradual que surge de la expe- 
riencia individual. Por ello, las teorías de estilos de aprendizaje se han convertido en una alternativa para explicar el motivo por el cual en un mismo grupo, los estudiantes aprenden de manera diferente y las estrategias de aprendizaje sirven para facilitar la adquisición, almacenamiento y/o uso de la información ${ }^{3,4}$.

El aprendizaje es un proceso de construcción individual y social, que el estudiante debe regular y por el que tiene que responsabilizarse. Es decir debe promoverse un aprendizaje autónomo. Biggs, propone cuatro condiciones que generarían un aprendizaje de calidad en el estudiante universitario: una base de conocimientos bien estructurada, un contexto motivacional adecuado, actividad por parte del estudiante y la interacción con otros. Es necesario resaltar la estrecha relación entre el aprendizaje y el rendimiento académico, asimismo referir que el rendimiento académico es un resultado del aprendizaje $e^{4}$.

Se ha evidenciado que para los estudiantes el aprendizaje es eminentemente memorístico, no son estratégicos, por lo tanto su rendimiento académico es deficiente. En Argentina, se constató que varios estudiantes al ingresar utilizan estrategias defectuosas de mecanización memorística, siendo carentes de metacognición, generando un comportamiento desprovisto de autorregulación y con dificultades en la aplicabilidad del conocimiento en situaciones nuevas. Razón por la cual los estudiantes están más motivados por evitar el fracaso que por aprender, estudiar para aprobar en lugar de estudiar para saber o aprender. El aprendizaje superficial suele ser característico en muchos de estos estudiantes, se ha demostrado que utilizan este tipo de aprendizaje y recurren a concentrarse en estrategias de memorización y retención, encuentran el trabajo pesado e ingrato, y suelen fracasar con mayor frecuencia en los exámenes; mientras que los estudiantes que optan por un aprendizaje profundo emplean más tiempo en el estudio y consideran el material que aprenden como más fácil de comprender ${ }^{3,6,7,8}$.

En el caso de los estudiantes universitarios "el rendimiento académico apropiado, puede hacer referencia a aspectos muy diversos, entre los cuales destacan, terminar un semestre con todas las asignaturas aprobadas, no repetir semestres o asignaturas, no abandonar la carrera, aprender de a verdad, realizar todos los exámenes y aprobarlos". Condición que no se observa en los estudiantes de bajo rendimiento académico. Por estas razones el uso de estilos de aprendizaje juega un papel determinante en la formación académica de los estudiantes de nivel superior ${ }^{6,7}$.

Beltrán ${ }^{8}$ define las estrategias de aprendizaje como actividades u operaciones mentales que se emplean para facilitar la adquisión de conocimientos. Se caracterizan porque son manipulables directa o indirectamente, además de tener un carácter intencional ${ }^{8}$.

El docente debe asumir un rol activo y preventivo conjuntamente con su Institución, lo que implica que su actuar académico, debe propiciar el aprendizaje de cada uno de los estudiantes; atendiendo las diferencias individuales en los estilos y estrategias de aprender, bajo este sustento se requiere conocer e intervenir en esta área ${ }^{5}$ Los estilos sirven como indicadores estables de como los estudiantes interactúan y responden a sus ambientes de aprendizaje. Mientras las estrategias son un proceso de toma de decisiones consciente e intencional sobre que conocimientos (especialmente los procedimentales) va a utilizar para alcanzar determinados objetivos de aprendizaje ante determinadas situaciones educativas a enfrentar; lo que exige toma de decisiones para tener la capacidad de reflexionar sobre cuándo y porqué debe emplearse (un conocimiento conceptual o actitudinal) para lograr un aprendizaje, lo cual va más allá de una simple acción automática y rutinaria de un conjunto de técnicas ${ }^{9,10,11}$

El conocimiento del estilo y estrategia de aprendizaje predominante en las aulas universitarias es una herramienta útil para adaptar el estilo de enseñanza del docente, para estimular un mejor rendimiento; a la vez permite un mejor monitoreo y evaluación del aprendizaje. Asimismo es de mucha utilidad para el estudiante porque le permite planificar su aprendizaje según sus estilos y estrategias evitando los bloqueos y optimizando resultados ${ }^{3}$. La Facultad de Odontología está comprometida en la búsqueda de la calidad educativa, enfrentando altos índices de deserción, abandono y/o rezago estudiantil en los primeros años de estudios, por lo que se hace necesario investigar en este ámbito.

La presente investigación tiene como objetivo identificar las estrategias, los estilos de aprendizaje en los estudiantes ingresantes en la Facultad de
Odontología y su relación con el rendimiento académico.

\section{Materiales y métodos.}

El presente estudio es cuasi experimental, cuanticualitativo, comparativo.

La población de estudio: estudiantes ingresantes

Ubicación en el espacio y tiempo: Facultad de Odontología-UNMSM- de mayo a setiembre 2013

Las unidades de observación lo constituyen 26 estudiantes que participaron en forma voluntaria en la convocatoria al Programa de Tutoría, el que tuvo dificultades de implementación.

Criterios de inclusión: estudiantes matriculados con asistencia regular.

Se solicitó previamente consentimiento informado a los estudiantes y docentes que participaron en el estudio.

Esta investigación tuvo tres fases:

Primera Fase: Se identificaron los estilos y estrategias de aprendizaje iniciales de los estudiantes ingresantes y su perfil sociodemográfico.

Segunda Fase: Se realizaron tres encuentros, dos para información general y otra personalizada sobre estilos y estrategias de aprendizaje, el primero entre docentes tutores, el segundo con los docentes tutores y con los estudiantes y el tercero con los tutores y cada uno de los estudiantes. No se realizó el acompańamiento sostenido a los estudiantes porque hubo dificultades de implementación del Programa de Tutoría.

Tercera Fase: Se evaluó los estilos y estrategias de aprendizaje y la relación con su rendimiento académico.

Se utilizó el Cuestionario Honey-Alonso de Estilos de Aprendizaje (CHAEA) para identificar los estilos de aprendizaje: Activo, Reflexivo, Teórico y Pragmático; consta de 80 preguntas cuya respuesta (más) significa de acuerdo total y - (menos) en menor acuerdo, se encuentran divididas en cuatro secciones de veinte preguntas cada una, las que se refieren a los cuatro estilos de aprendizaje. El nivel de confiabilidad es considerado como aceptable y cuenta con validez de constructo ${ }^{1}$.

Las estrategias de aprendizaje se evaluaron utilizando el cuestionario de evaluación de las estrategias de aprendizaje de los estudiantes universitarios, de Gargallo². El que tiene 88 ítems, con respecto a los cuales el sujeto se pronuncia en una escala tipo Likert 
de 5 grados (Acuerdo-Desacuerdo), en función de la valoración y/o uso del ítem correspondiente de acuerdo a la estrategia de que se trate. Involucran el uso de las estrategias afectivas de apoyo y de control (automanejo) como las estrategias de procesamiento de la información ${ }^{2}$.

El rendimiento académico se evaluó con el historial académico, teniendo en cuenta la aprobación o no de alguna de las 7 asignaturas.

\section{Resultados}

Se caracterizó el perfil del estudiante, siendo su edad promedio de 18.80 años con una desviación estándar de 2,23; el $42 \%$ es femenino y el $58 \%$ masculino; en cuanto a la Institución Educativa de procedencia el $22 \%$ es Estatal y el $78 \%$ de Particular.

En cuanto a las estrategias de aprendizaje iniciales, se encontró que la valoración de la importancia de la planificación para el aprendizaje un 55\% refería estar muy de acuerdo y de acuerdo, así como en tener un horario de trabajo para el estudio personal; en cuanto a las habilidades de interacción social y trabajo con compañeros para estudiar o realizar trabajos en grupo un 60\% refirió estar muy de acuerdo y de acuerdo; mientras que si él escoge a compañeros adecuados para el trabajo en equipo un $60 \%$ está muy de acuerdo y de acuerdo, siendo un $40 \%$ los indecisos y en desacuerdo. En cuanto al control del contexto el $40 \%$ refiere estar indeciso para aprovechar su tiempo para estudiar; en cuanto a si trabaja y estudia en un lugar adecuado un 52\% refiere estar muy de acuerdo y de acuerdo. En cuanto al tener conocimientos sobre el procesamiento de la información y al tener acceso a fuentes de información, en manejo de la biblioteca y hemeroteca el 60\% está indeciso.

En cuanto a selección de información en relación a la capacidad de separar información fundamental de la que no lo es un $40 \%$ refiere estar indeciso, siendo igual para la organización de la información en gráficos, esquemas, tablas. Mientras que en cuanto a la personalización y creatividad, en relación a temas que estudia como a la capacidad de aportar un $40 \%$ es indeciso; mientras que en el manejo de recursos para uso eficaz de la información hay un $45 \%$ de indecisos.

Se identificó que al inicio de sus estudios la motivación intrínseca era calificada como importante y necesaria con la categoría de muy de acuerdo el 60\% y de acuerdo el $40 \%$, mientras que al término el $39 \%$ refería estar muy de acuerdo y el $51 \%$ de acuerdo. En cuanto a las atribuciones internas disminuye de un $55 \%$ de muy de acuerdo a un $38 \%$; mientras que las atribuciones externas se incrementan de un 39\% a un $52 \%$ como muy de acuerdo. En la concepción de la inteligencia como modificable existe un decrecimiento de un $51 \%$ de muy de acuerdo a un $47 \%$.

En relación al control de la ansiedad existe un decrecimiento de un 54\% como de muy acuerdo a un $42 \%$.

A la pregunta si conoce los objetivos y los criterios de evaluación de las asignaturas que cursa, al inicio el $9 \%$ refiere estar de acuerdo, y un $60 \%$ en desacuerdo, teniendo al final un 30\% respectivamente. (Ver Graf. 1).

Graf.1. Conocimiento de objetivos y criterios de evaluación de las

asignaturas que cursa

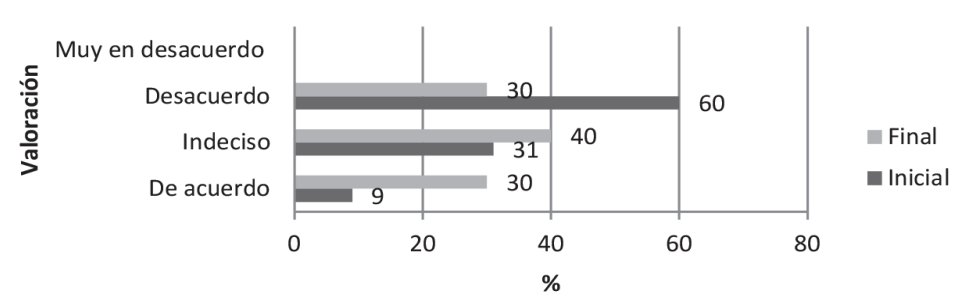

A la pregunta si planifica su tiempo para trabajar a lo largo de sus asignaturas el 55\% refiere estar de muy acuerdo al inicio, siendo el final de un $45 \%$. (Ver Graf, 2).

Graf 2. Planifico mi tiempo para trabajar a lo largo de las asignaturas

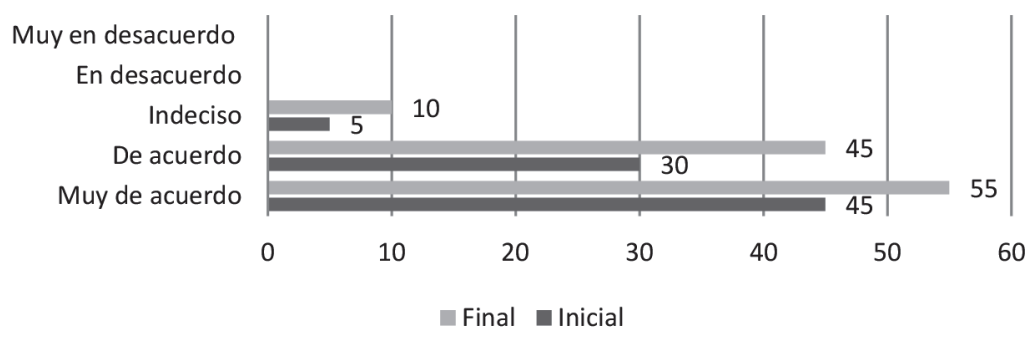

En cuanto al estilo de aprendizaje inicial el 13\% refiere tener de preferencia el activo, siendo el 25\% reflexivo, el 44\% teórico y el 19\% pragmático; mientras que el estilo de aprendizaje de preferencia final es el teórico con un 81\%. (Ver Grá.3).

Graf.3. Estilos de aprendizaje inicial y final

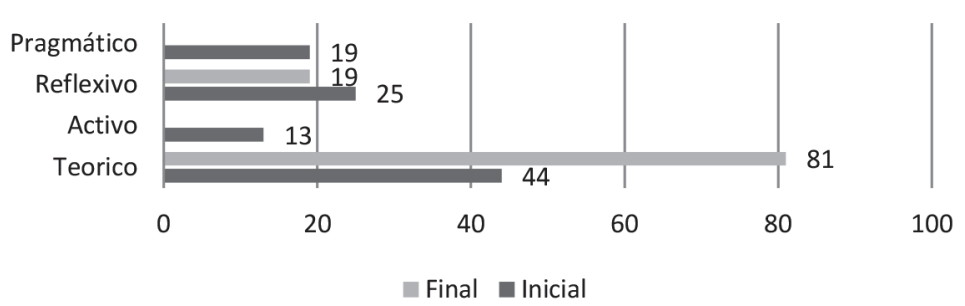


El rendimiento académico se valoró con las calificaciones obtenidas, siendo el $80 \%$ de estudiantes aprobados.

Se encontró una diferencia significativa al comparar la estrategia de aprendizaje tomando en cuenta la motivación al inicio y final de su año académico, al hacer el análisis de contraste de muestras relacionadas con la prueba de Mac Nemar, entre la motivación extrínseca inicial y final con un valor de $\mathrm{p}<0.008$, a un nivel de significancia de 0.05 (Tabla 1).

Tabla 1. Comparación de la motivación extrínseca inicial y final

\begin{tabular}{|c|c|c|c|}
\hline Hipótesis nula & Test & $\begin{array}{l}\text { Sig. Asin- } \\
\text { tótica }\end{array}$ & Decisión \\
\hline $\begin{array}{l}\text { Las distribucio- } \\
\text { nes de valores } \\
\text { diferentes entre } \\
\text { Motivación } \\
\text { para el aprendi- } \\
\text { zaje final y Mo- } \\
\text { tivación para } \\
\text { el aprendizaje } \\
\text { inicial tienen } \\
\text { las mismas } \\
\text { probabilidades }\end{array}$ & $\begin{array}{l}\text { Prueba } \\
\text { Mc Nemar } \\
\text { de muestras } \\
\text { relacionadas }\end{array}$ & .008 & $\begin{array}{l}\text { Rechazar } \\
\text { la hipotesis } \\
\text { nula }\end{array}$ \\
\hline
\end{tabular}

Se encontró una diferencia significativa al comparar la estrategia de aprendizaje motivación para el aprendizaje con el rendimiento académico al inicio y al final del año académico, al hacer el análisis de contraste de muestras relacionadas con la prueba de Mac Nemar, con un valor de $\mathrm{p}<0.006$, a un nivel de significancia de 0.05 (Tabla 2).

Tabla 2. Comparación de la motivación y rendimiento académico, inicial y final

\begin{tabular}{|c|c|c|c|}
\hline Hipótesis nula & Test & $\begin{array}{l}\text { Sig. Asin- } \\
\text { tótica }\end{array}$ & Decisión \\
\hline $\begin{array}{l}\text { Las distri- } \\
\text { buciones de } \\
\text { Motivación para } \\
\text { el aprendizaje } \\
\text { final, Rendimiento } \\
\text { académico y } \\
\text { Motivación para el } \\
\text { aprendizaje inicial } \\
\text { son las mismas. }\end{array}$ & $\begin{array}{l}\text { Prueba } Q \text { de } \\
\text { Cochran de } \\
\text { muestras } \\
\text { relacionadas }\end{array}$ & .006 & $\begin{array}{l}\text { Rechazar } \\
\text { la hipote- } \\
\text { sis nula }\end{array}$ \\
\hline
\end{tabular}

Se encontraron puntuaciones altas pero no con diferencias estadísticamente significativas en las estrategias: atribuciones externas, estrategias de planificación, estrategias de selección de información, estrategias de elaboración de la información, organización y personalización.

Asimismo en el valor de la tarea, persistencia y atribuciones, autoeficacia y expectativas; concepción de la inteligencia como modificable; planificación, control y autorregulación; adquisición de la información, elaboración de información, Organización, información, personalización y creatividad, pensa- miento crítico, almacenamiento, manejo de recursos y transferencia para uso eficaz de la información; control del contexto, habilidades de interacción social y trabajo con compañeros; conocimientos de fuentes y búsquedas de información, selección de información.No se encontró relación entre género y estrategias metacognitivas.

\section{Discusión}

En la Universidad se espera que los estudiantes a su ingreso sean competentes, autorregulados, activos, con un enfoque profundo del aprendizaje; la realidad demuestra lo contrario, esta condición no es plena, pues se han visto influidos por las condiciones y características del contexto que determina su aprendizaje, de los contenidos, del tipo de evaluación, entre otros.

La motivación intrínseca y la ausencia de ansiedad, se asocian con el enfoque profundo para aprender. Mientras que la motivación extrínseca, la ansiedad y la ausencia de una motivación intrínseca se correlacionan igualmente con un enfoque superficial ${ }^{3,4,6}$.

Una estrategia, es el conjunto de decisiones que se toman de manera ajusta$\mathrm{da}$ a las condiciones del problema que se intenta resolver, y una estrategia de aprendizaje es una decisión consciente en función de la demanda de la tarea a realizar y de las condiciones personales, en especial del conocimiento de los propios recursos con que cuenta el discente para enfrentar esa demanda. En esta descripción hay dos elementos importantes, primero, que toda estrategia supone una toma de decisiones, que posibilita una planificación por parte del sujeto tanto de las objetivos como del modo de alcanzarlos en función de factores internos y externos a la propia tarea; el segundo elemento se refiere al hecho de que toda estrategia de aprendizaje supone de parte del discente un conocimiento tanto de cómo se ejecuta la técnica como de saber cuándo y por qué es útil la utilización de dicha técnica. A esto último se le denomina conocimiento estratégico ${ }^{3,4,6}$.

El presente estudio ha podido evidenciar que el estudiante ingresante aún está en proceso de fortalecer su autonomía, se encontraron diferencias estadísticamente significativas en las estrategias motivacionales, donde la amenaza de las atribuciones externas se hace evidente con el incremento de la motivación extrínseca para su aprendizaje; lo que los acerca al uso de estrategias superficiales para aprender.

Las estrategias de aprendizaje son procesos de toma de decisiones que se realizan conscientemente para alcanzar un objetivo, en los que se activan técnicas y procedimientos de distinta naturaleza (disciplinarios e interdisciplinarios). La noción de especificidad da a entender que el abordar diferentes tipos de tareas supone el desarrollo de diferentes maneras o procedimientos con respecto a la naturaleza de las tareas que se van a realizar. Ese punto de vista que destaca la especificidad en la manera como se reacciona frente a las exigencias de aprendizaje, nos lleva a considerar la cuestión de la variabilidad de las estrategias; lo que a su vez nos conduce a una distinción conceptual importante: la estrategia y el estilo de aprendizaje $12,13,14$

Es necesario hacer una clara distinción entre los conceptos de "estrategia", "estilo" de aprender; se utilizan de manera indiferenciada para definir la problemática relativa a lo que hacen los estudiantes cuando enfrentan un contenido de estudio. Sin embargo, algunos autores introducen precisiones en esos conceptos haciendo alusión al carácter permanente, intencional o a la asociación con una fuerte base motivacional. El primer concepto, estrategia, se muestra más específico o particular, respondiendo a una situación determinada; mientras que el segundo, estilo, aparece como más general y constante, respondiendo más a una tendencia del sujeto. En este sentido, el estilo sería la disposición o inclinación de un individuo a adoptar la misma estrategia en diversas situaciones. Como se deduce de este análisis, la estrategia de aprendizaje es subsidiaria del estilo de aprendizaje $\mathrm{e}^{14,15,16}$.

Juárez ${ }^{17}$, identificó que en los estudiantes de primer semestre predomina el estilo reflexivo, mientras que el presente estudio encontró que prevalece el teórico tanto al inicio como al final. Ambos estudios no hallaron relación entre los estilos de aprendizaje con el rendimiento académico de los estudiantes ingresantes. Mientras que Madrid $^{13}$; encontró que predominaba el estilo pragmático. Se hace necesario proporcionar mejores oportunidades de aprendizaje diseñando actividades y tareas que favorezcan el desarrollo de todos los estilos para así darles la oportunidad a todos los estudiantes de transformar y potenciar sus capacidades de aprender. 
El valor de la tarea puede conducir al estudiante a involucrarse más con su proceso de aprendizaje ${ }^{17,18}$; los resultados en el presente estudio de esta subescala indican que no hay diferencia significativa en el valor que otorgan los estudiantes a la tarea.

Morera $^{18}$ en España 2010; encontró diferencias estadísticamente significativas en las estrategias integrantes de la primea subescala; donde disminuye la motivacion intrínseca y se incrementa la motivacion extrínseca, coincidiendo con el presente estudio. También halló diferencias estadísticamente significativas en las estrategias de procesamiento de la información, lo cual no coincide con los resultados del presente estudio.

Juárez ${ }^{17}$; identificó que los cuestionarios de Estilos de Aprendizaje (CHAEA) y la Escala de Estrategias de Aprendizaje, son herramientas potenciales que el tutor puede emplear para identificar las características psicoeducativas de los estudiantes tutorados que ingresan a una universidad pública de México; encontró correlación significativa entre las variables estrategias y estilos de aprendizaje con el promedio final del primer semestre; el presente estudio encontró diferencias significativas entre una de las estrategias, la motivación en relación al rendimiento académico; permitió diferenciar las potencialidades de los estudiantes en cuanto al uso de estrategias para el aprendizaje coincidiendo con el estudio del autor mencionado.

Es importante promover en las aulas universitarias las diversas estrategias de aprendizaje, puesto que está comprobado, a través de diferentes investigaciones, que la mayoría de los estudiantes no utilizan estrategias adecuadas para lograr un aprendizaje significativo. El conocimiento de las estrategias de aprendizaje empleadas por los estudiantes y la promoción de aquellas que favorezcan el rendimiento, permitirá también el entrenamiento en su uso para aquellos sujetos que no las desarrollan o que no las aplican de forma efectiva, mejorando así sus posibilidades de trabajo y estudio ${ }^{18}$.

Díaz ${ }^{19}$ identificó en la Universidad Técnica del Callao, que los estudiantes de primer año tienen un predominio a su ingreso del estilo de aprendizaje teórico (51\%), seguido del activo (21\%); mientras que el presente estudio encontró que prevalece el teórico (44\%), seguido del reflexivo (25\%) al inicio, continuando el teórico al final en un (81\%).

\section{Conclusiones:}

La estrategia de aprendizaje motivacional es la que predomina en los estudiantes ingresantes, donde la amenaza de las atribuciones externas se evidencia con el incremento de la motivación extrínseca para su aprendizaje, siendo significativo a la relación con el rendimiento educativo. Lo cual indica que si los contextos de aprendizaje no cambian, el estudiante a medida que avanza en sus estudios se desmotiva y se vincula sólo a responder a lo que el contexto le propone y se refleja en su rendimiento.

Es ineludible acercar al estudiante a experiencias de aprendizaje que movilicen el desarrollo de sus potencialidades

El estilo de aprendizaje que predomina en el estudiante ingresante al iniciar y al finalizar sus estudios universitarios, es el teórico. Lo que describe que el estudiante ingresante aún está en proceso de la construcción de su autonomía y requiere orientación.

La comunicación entre docentes y estudiantes requiere ser permanente, de tal manera que el estudiante tenga claro los objetivos a lograr y sus formas de evaluación.

Fortalecer la autonomía en el aprendizaje del estudiante, es una necesidad y se logra incidiendo en el desarrollo del estilo de aprendizaje reflexivo, el que permite equilibrar el estilo teórico.

La aplicación de las estrategias metacognitivas dependen de la institución formadora, igual que la motivación.

Las estrategias de aprendizaje tienen relación con el rendimiento académico de los estudiantes ingresantes.

\section{Recomendaciones:}

Es necesario hacer estudios longitudinales y con mayor muestra de sujetos de estudio, lo que permitirá comprender mejor los procesos de aprendizaje y sus logros.

Es necesario continuar esta línea de investigación en todos los años de estudios, para fortalecer capacidades en los estudiantes y docentes.

Es ineludible continuar con estudios relacionando estrategias y estilos de aprendizaje, incorporando los estilos de enseñanza.

Establecer una evaluación permanente de las estrategias de aprendizaje, estilos de aprendizaje y rendimiento académico involucrando satisfacción, bienestar y éxito académico por año académico.
Realizar un diagnóstico triangulando: el historial académico, caracterizando los estilos de aprendizaje y las estrategias, sumados a sus percepciones (cualitativo) de todos los estudiantes de primer año.

Con los resultados obtenidos se propone hacer un monitoreo y retroalimentación, acción-observación-reflexión -evaluación y nueva acción.

\section{Agradecimiento}

Al Vicerrectorado de Investigación que permitió el financiamiento del estudio y al equipo de docentes tutoras por su compromiso.

\section{Referencias bibliográficas.}

1. Alonso CM, Honey P. Los estilos de aprendizaje. Procedimientos de diagnóstico y mejora. Revista estilos de aprendizaje. (Internet). 2012 (consultado: 01 de enero del 2013);10(19:1-14. Disponible en: http://www.estilosdeaprendizaje.es/ chaea/chaea.htm.

2. Gargallo B. et al. El cuestionario CEVEAPEU. Un instrumento para la evaluación de las estrategias de aprendizaje de los estudiantes universitarios. RELIEVE (Internet). 2009 (Consultado el 3 de mayo de 2012);15(2),1-31. Disponible en: http://www.uv.es/RELIEVE/ v15n2/RELIEVEv15n2_5.htm

3. Lopez M, López A. Los enfoques de aprendizaje. Revisión conceptual y de investigación. Revista Colombiana de Educación, primer semestre. 2013; 64

4. Biggs, J. Learning Process Questionnaire (LPQ). Manual. Hawthorn: Australian Council for Educational Reseach. 1987

5. Melare D. La función Tutorial en el proceso de formación continua docente. RIED (Internet). 2009. (Consultado el 02 de diciembre del 2011). Disponible en:http:// www.utpl.edu.ec/ried/images/pdfs/ Volumen12N1/ried12-1.pdf.

6. Amaya J, Prado E. Estrategias de aprendizaje para universitarios un enfoque constructivista. México: Trillas. 2002.

7. Barca A, Peralbo M, Porto AM, Santorum R, Vicente F. Estrategias de aprendizaje, autoconcepto y rendimiento académico en la adolescencia. Portugal, Espańa: Acta X Congreso Internacional GallegoPortugues de Psicopedagogía. 2009. 
8. Beltrán J. Estrategias de aprendizaje. Revista de educación 2003;332:55-73.

9. González D, Díaz Y. La importancia de promover en el aula estrategias de aprendizaje para elevar el nivel académico en los estudiantes de psicología. Revista Iberoamericana de Educación. 2006;40(1). Consultado el 02 de diciembre del 2012. Disponible en:http://www.reoie.org/ investigacion/137gonzalez.pdf.

10. Martín E, García L, Torbay A, Rodríguez T. Estrategias de aprendizaje y rendimiento académico en estudiantes universitarios. Int J Psychology and Psychological Therapy (Internet) 2008 (Consultado 02 de diciembre del 2012);(8)3:401-412. Disponible en: http://acapsi.com/ estrategias_de_aprendizaje_y_ rendimiento_academico_en_ estudiantes_universitarios.html.

11. Honey P, Mumford A. The Manual of Learning Styles. Maidenhead, Berkshire: Ardingly House. 1986.

12. Mead S. The evidence suggests otherwise: The truth about boys and girls. Education Sector (Internet) 2006 (Consultado: 02de diciembre del 2012). Disponible en: http:// www.educationsector.org/sites/ default/files/publications/ESO_ BoysAndGirls.pdf

13. Román JM, Gallego S. Escala de estrategias de aprendizaje. ACRA.
Madrid; TEA Ediciones. Schunk, D. H. Enhancing Self Efficacy and Achievement Trough Reward and Goals: Motivational and Informational effects. Journal of Educational Research 1984;78:2934

14. Juarez C. Rendimiento académico y estilos de aprendizaje en estudiantes de Psicología. Revista Estilos de Aprendizaje (Internet) 2011, abril (Consultado: 02 de diciembre del 2012); 7(7). Disponible en: http://www.uned. es/revistaestilosdeaprendizaje/ numero_7/sumario_completo/ 1sr_7_abril_2011.pdf.

15. Madrid V, et al. Perfil de estilos de aprendizaje en estudiantes de primer año de dos carreras de diferentes Áreas en la Universidad de Concepción. Revista Estilos de Aprendizaje. (Internet) 2009 (Consultado: 02 de diciembre del 2012); 3(3). Disponible en; http://www.uned. es/revistaestilosdeaprendizaje/ numero_3/artigos/1sr_ 3 _ abril_2009.pdf.

16. Fuentes A, et al. Escala de estrategias de aprendizaje ACRA. Abreviado para alumnos universitarios. Revista electrónica de Investigación Psicoeducativa y psicopedagógica. (Internet) 2003. (Consultado el 04 de diciembre del 2012);1(2).
Disponible en: http://www. investigacionpsicopedagogica.org/ revista/new/ContadorArticulo. php?16

17. Juárez L, et al. El cuestionario de estilos de aprendizaje CHAEA y la escala de estrategias de aprendizaje ACRA como herramienta potencial para la tutoría académica. Revista Estilos de Aprendizaje. (Internet) 2012 (Consultado el 04 de diciembre del 2012);10(10). Disponible en: http://www.uned. es/revistaestilosdeaprendizaje/ espanol/articulos.html\#a

18. Morera B, et al. Metodología innovadora y estrategias de aprendizaje en la Universidad. España (Internet) 2010. (Consultado el 04 de diciembre del 2012). Disponible en: http:// web.ua.es/es/ice/jornadas-redes/ documentos/2013/334820.pdf

19. Díaz A. Relación entre los Estilos de Aprendizaje y el Rendimiento Académico de las Estudiantes de la Escuela Profesional de Enfermería de la Facultad de Ciencias de La Salud de La Universidad Nacional Del Callao. (Internet) 2012. (Consultado el 02 de diciembre del 2012). Disponible en:http://www.unac.edu.pe/ documentos/organizacion/vri/cdcitra/ Informes_Finales_Investigacion/IF_ NOVIEMBRE 2012/IF_DIAZ\%20 TINOCO_FCS/INFORME $\% 20$ DE\%20INVESTIGACION.pdf 